\title{
Germanica
}

\section{« Ein Verein von Betonköppen, die sich die Hose mit der Kneifzange anziehen... »}

Die Generation 89 und die Stasi

«Ein Verein von Betonköppen, die sich die Hose mit der Kneifzange anziehen...»

Die Generation 89 und die «Stasi»

\section{Michael Klees}

\section{(2) OpenEdition}

Journals

Édition électronique

URL : http://journals.openedition.org/germanica/351

DOI : 10.4000/germanica.351

ISSN : 2107-0784

Éditeur

Université de Lille

Édition imprimée

Date de publication : 1 décembre 2006

Pagination : 215-227

ISBN : 2-913857-18-3

ISSN : 0984-2632

Référence électronique

Michael Klees, « « Ein Verein von Betonköppen, die sich die Hose mit der Kneifzange anziehen... » », Germanica [Online], 39 | 2006, Online erschienen am: 19 Februar 2010, abgerufen am 06 Oktober 2020. URL : http://journals.openedition.org/germanica/351 ; DOI : https://doi.org/10.4000/germanica.351

Ce document a été généré automatiquement le 6 octobre 2020.

(c) Tous droits réservés 


\title{
« Ein Verein von Betonköppen, die sich die Hose mit der Kneifzange anziehen... $»^{1}$
}

\author{
Die Generation 89 und die Stasi
}

«Ein Verein von Betonköppen, die sich die Hose mit der Kneifzange anziehen...»

Die Generation 89 und die «Stasi»

\section{Michael Klees}

\section{Vorbemerkung}

1 Etwa mit dem Erscheinen von Ingo Schulzes Simple Storys ${ }^{2}$, spätestens aber mit Christoph Heins Willenbrock ${ }^{3}$ schien es, als habe sich die Wendeliteratur bzw. die Literatur zur Deutschen Einheit selbst überholt, zumindest aber eine weitere, neue Qualität angenommen. In einer Spiegel-Rezension nennt Wolfgang Höbel Schulzes Buch, das den Untertitel «Geschichten aus der ostdeutschen Provinz » trägt, «den lang ersehnten Roman über das vereinigte Deutschland" ${ }^{4}$. Tatsächlich beschreiben 29 Geschichten, lose über ihre Protagonisten verbunden, emotionslos Unspektakuläres aus dem thüringischen Altenburg und der Umgebung. Die Fabel behandelt jeweils das Alltägliche: Die Busgruppenreise als kleine Flucht vor der Realität, Arbeitslosigkeit, Liebesglück und -unglück, Autounfälle, aufkeimenden Rechtsextremismus usw. Zwar spielt sich alles noch auf der Folie des von der Wiedervereinigung betroffenen und gezeichneten Ostens ab, die tatsächlichen Probleme der geschilderten Personen sind allerdings längst im gesamtdeutschen Alltag angekommen und selbst da, wo sie auf originäre Ereignisse aus der DDR-Vergangenheit zurückgeführt werden, haben sie anders als in vorausgegangenen Auseinandersetzungen anderer Autoren mit DDR und Wiedervereinigung, wo es häufig um Fragen nach Identität, Schuld oder Zukunftsperspektiven geht - ihre Relevanz verloren. Beispielhaft mag jene Szene gelten, in der während der schon angedeuteten Busreise nach Italien, der zu DDRZeiten als Regimekritiker entlassene, ehemalige Lehrer Schubert den verantwortlichen 
Rektor seiner alten Schule, Ernst Meurer, erkennt und diesen in der öffentlichkeit als Funktionär bloßzustellen versucht. Wie die Erzählerin, Meurers Frau Renate, beschreibt, wird dies jedoch zur Blamage für Schubert selbst:

Natürlich wusste keiner, wer gemeint war, als er vom «roten Meurer» sprach. Die Italiener verstanden ihn sowieso nicht. Er nannte Ernst den «Bonzen in dem grünen Anorak» und wies mit ausgestrecktem Arm auf uns. Keiner Begriff, was er wollte. Vor allem wunderte ich mich, woher er die Kraft nahm zu schreien, so aufgebracht zu schreien. Die Geschichte lag weit zurück. Und Gern hat es Ernst damals nicht gemacht, das weiß ich. ${ }^{5}$

Während die Aktion für Schubert im Gewahrsam der Carabinieri endet, geht für Renate und Ernst Meurer die Reise ungestört mit schönen Erinnerungen an alte Zeiten weiter. Fragen nach Schuld und Verantwortung verlieren völlig an Bedeutung, während für die Betroffenen und die Mitreisenden nur die Organisation der Zukunft zählt, in diesem Fall die ungestörte Weiterführung der Urlaubsfahrt.

Willenbrock, den Uwe Wittstock Heins «schönsten und reifsten Roman» ${ }^{6}$ nennt, geht noch einen Schritt weiter. Der desillusionierte, jeglicher Utopie beraubte Protagonist gleichen Namens ist längst im gesamtdeutschen Alltag angekommen. Statt $\mathrm{zu}$ resignieren, hat er nach dem Zusammenbruch der DDR einen erfolgreichen Gebrauchtwagenhandel aufgezogen, beschäftigte illegal einen kostengünstigen polnischen Mechaniker und machte beste Geschäfte mit russischen und osteuropäischen Partnern. Auf die gesamtgesellschaftliche Dimension bezogen, zeichnet sich hier - neben allgemeinen gesellschaftlichen Auflösungserscheinungen weitsichtig die wesentlich relevantere Ost-West-Problematik im europäischen Rahmen ab. Auf der persönlichen Ebene hat der Protagonist Willenbrock an seiner Vergangenheit nur noch ein beiläufiges, unbeteiligtes Interesse. Als er auf einer politischen Veranstaltung nach Jahren zufällig Feuerbach, jenen ehemaligen Gewerkschaftsvertrauensmann wieder trifft, der ihm wegen vermeintlicher politischer Unzuverlässigkeit seine einstige wissenschaftliche Karriere zerstört hat, empfindet er weder Hass noch Rachebedürfnis, statt dessen ist ihm die Situation schlicht unangenehm und er schlägt Feuerbach vor "Wollen wir so tun, als sei nichts gewesen? $\gg^{7}$ Später, als Feuerbach, wiederum zufällig, ausgerechnet bei ihm seinen alten Opel Kadett verkaufen will, fühlt sich Feuerbach von Willenbrocks Anteilnahme an seiner desolaten finanziellen Situation provoziert, worauf hin er seinerseits Willenbrock herausfordert, ihn zur Rede zu stellen, bis es zu Tätlichkeiten kommt ${ }^{8}$. Letztlich jedoch

tat ihm [Willenbrock, M.K.] Feuerbach Leid, nicht, weil er ihn mit seiner Faust ins Gesicht geschlagen hatte, sondern weil er doch im Grunde ein armes, lächerliches Würstchen war, ein ewig zu kurz gekommener, der solche Berichte nur verfasste, weil sie ihm scheinbar eine Macht und Bedeutung gaben, die ihm in seinem Leben und bei seiner Arbeit fehlten'.

4 Am liebsten wäre ihm die Auseinandersetzung mit Feuerbach erspart geblieben, so irrelevant und fern ist die Vergangenheit inzwischen für Willenbrock.

Einer der wichtigsten Motiv-Komplexe in der Literatur zur Deutschen Einheit ist unzweifelhaft die Auseinandersetzung mit der «Stasi», dem Ministerium für Staatssicherheit (MfS) sowie deren Machenschaften. Die Feuerbach-WillenbrockVerbindung scheint dies im Spitzel-Motiv aufzugreifen, bleibt in der Konsequenz allerdings bedeutungslos und kontrastiert statt dessen die Aktualität der anderen erzählen Ereignisse. Für die Simplen Storys gilt ähnliches. Zwar kommen Motive im 
Zusammenhang mit der Stasi noch vor, allerdings treten sie als bedeutungslose, aber kuriose und dementsprechend feinsinnig-ironisch gekennzeichnete Marginalien in den Hintergrund: «Ich sehe auf die Holzfolie seines Stasi-Tisches - das Mobiliar der örtlichen Staatssicherheit war der "Lebenshilfe e.V." übergeben worden, und die hatten, was sie nicht brauchten, weiter verkauft - alles billiger Krempel $»^{10}$. - So wird die Stasi zum Ramschladen der Geschichte.

Wäre man nun aber angesichts der an Schulze und Hein aufgezeigten Tendenz davon ausgegangen, dass die literarische Auseinandersetzung mit DDR, Wende, Wiedervereinigung und einhergehenden Problemen abnehmen und aus dem Publikumsinteresse verschwinden würde, hätte man sich durch den überaus großen Erfolg von Jana Hensels Zonenkindern ${ }^{11}$ und die folgende Flut von Erinnerungsbüchern eines Besseren belehren lassen müssen. Die Literatur zur Deutschen Einheit ist damit in eine neue Phase eingetreten.

7 Im folgenden soll an einigen Vertretern dieser neuen Generation untersucht werden, ob auch hier die - in den literarischen Phasen zuvor so bedeutende - Stasi-Motivik noch eine Rolle spielt und wenn ja, welche.

\section{Zonenkiner}

8 Jana Hensels autobiographisch-dokumentarisches Buch hat sich bereits im ersten Jahr seines Erscheinens über 160000 Mal verkauft und legte dank des immensen Publikumszuspruchs den Grundstein für eine neue Autorinnen- und Autorengeneration, vom Feuilleton in Anlehnung an Florian Illies Bestandsaufnahme Generation Golf ${ }^{2}$ gerne als «Generation Wartburg» oder «Generation Trabbi» bezeichnet. Gemeint ist eine junge, ostdeutsch sozialisierte, aber zum großen Teil im vereinigten Deutschland aufgewachsene Generation von Schreibenden. Jana Hensel selbst charakterisiert die Situation der «Generation 89» in dem für ihr Buch so typischen «wir» - das später ein Hauptanstoß der Kritik werden sollte - so:

Die paar Jahre vor dem Fall der Mauer, die wir dort gelebt haben, machen zurzeit noch die Hälfte unseres Lebens aus. Von nun an werden sie jedoch zahlenmäßig in die Minderheit geraten und die DDR für uns, als schauen wir in den Rückspiegel eines Autos, noch ferner, kleiner und immer märchenhafter werden. ${ }^{13}$

9 Wesentliches Merkmal dieser in der Regel um die Mitte der 70-er Jahre Geborenen ist, dass sie in beiden Systemen zu Hause sind: im Osten ihre Kindheit verbracht haben und sich nach der Wende am Westen justieren mussten, und dies oftmals sehr erfolgreich.

Wie aber erklärt sich nun das überproportionale Leserinteresse an den Zonenkindern bzw.den Texten der 89-er allgemein? Den Erfolg einfach nur auf eine neue OstalgieWelle zu schieben, wäre sicher zu kurz gegriffen, ist der Publikumszuspruch im Westen Deutschlands doch noch größer als im Osten. Dies belegen die Verkaufszahlen. Bereits 1990 hat Karl Heinz Bohrer, in der Diskussion um das weitere Schicksal der DDR, auf den «exotischen Reiz»" ${ }^{14}$ hingewiesen, den Ostdeutschland auf westdeutsche Besucher ausübe und vor Versuchen gewarnt, «die DDR als eine Art Naturschutzpark eines sozial und ökonomisch verträumten Gestern zu bewahren, an der die BRD ihr notwendig schlechtes Gewissen täglich erneuern kann» ${ }^{15}$. Der Lauf der Geschichte hat dies verhindert, allerdings liegt der Verdacht nahe, dass dieser von der Generation 89 beschriebene Osten auch noch oder gerade fünfzehn Jahre später die exotischvoyeuristischen Bedürfnisse des Lesepublikums in seiner Vorliebe für die Ästhetik des 
Hässlichen ${ }^{16}$, in seinem trivialen «die-sind-so-schön-anders» anspricht bzw. befriedigt. Folgerichtig fragt Susanne Messmer schon 2002, «Könnte es nicht so sein, dass die Westler sich mit Hilfe dieser neuen Flut von Büchern noch einmal so richtig schön den Osten als das Andere imaginieren? $»^{17}$

Das Andere dient den Westlesern durch Abgrenzung zur Vergewisserung der eigenen Identität, während es für die Ostleser ja das Eigene ist und die Möglichkeit zur Identifizierung bietet. Hierbei spielt es keine besondere Rolle, ob es sich um eine tatsächliche oder eine konstruierte ostdeutsche Wirklichkeit handelt. Vielmehr scheint es, je stärker die Ost-Klischees bedient, je mehr die bestehenden Mythen abgearbeitet werden, desto erfolgreicher vermarktet sich das Buch. Damit korrespondiert etwa auch die Äußerung von Thomas Krüger, dem seinerzeitigen Präsidenten der Bundeszentrale für politische Bildung anlässlich eines Treffens erfolgreicher junger Ostdeutscher, die längst im Gesamtdeutschen fest verankert sind: «Es gibt gar keine Generation 89. Das ist nur eine Masche, um Bücher zu verkaufen.»18

Dementsprechend waren die Kritikermeinungen geteilt, entgegen der meist freundlichen Aufnahme der Texte durch die Leserschaft. Wechselweise wurde den Zonenkindern die fehlende soziologische oder historische Dimension unterstellt bzw. vorgeworfen. Tom Kraushaar, der Herausgeber einer ersten Sammlung mit Sekundärtexten zu Hensels Buch, glaubt sogar zu erkennen: «aber besonders mit dem Erfolg erhöhten sich auch Ausmaß und Schärfe der Polemiken.» ${ }^{19}$

Dabei macht sich die Erzählerin der Zonenkinder eigentlich doch nur auf die Entdeckungsreise nach ihrer Herkunft, wie sie sagt:

Ich möchte wieder wissen, wo wir herkommen, und so werde ich mich auf die Suche nach den verlorenen Erinnerungen und unerkannten Erfahrungen machen, auch wenn ich fürchte, den Weg zurück nicht mehr zu finden.

14 Der pathetisch anmutende Nachsatz hat eine gewisse Berechtigung. Zwar beschäftigt sich das Buch autobiographisch mit einer persönlich erlebten und/oder stilisierten DDR-Vergangenheit. Bei genauerer Betrachtung scheinen diese Geschichten allerdings nur den Hintergrund $\mathrm{zu}$ bilden für das eigentliche Thema: die Alterität, die interkulturellen Transferenzen zwischen jungen Erwachsenen aus Ost und West in den Jahren nach der Wiedervereinigung und die daraus resultierenden Verständnis- und Identitätsprobleme der Erzählerin.

Von der Stasi ist dementsprechend nur mittelbar zu lesen, ohne dass der Name überhaupt fällt. Da der Topos der allgegenwärtigen Staatsmacht, die ihre Bürger nach Kräften ausspionierte und so von den Menschen im Alltag ein Höchstmaß an Vorsicht und Diskretion verlangte, hinlänglich bekannt ist, errät der Leser schnell, dass es sich um Stasi-Spitzeltätigkeit bzw. um die Gefahr des Verrats durch Stasi-IM (Inoffizieller Mitarbeiter) handelt. Etwa wenn die Erzählerin von einer Familienfeier berichtet:

Dann schwang sich einer der Väter zum Sprecher des Abends auf [...] als sei er der Anführer einer kleinen revolutionären Bewegung [...]. Wenn des Anführers Ehefrau ängstlich guckte, und den Zeigefinger an den Mund legte, wussten wir Kinder, dass jetzt der rechte Zeitpunkt gekommen war, genauer hinzuhören. ${ }^{20}$

Der warnende Zeigefinger signalisiert die Furcht, dass die Kinder am nächsten Tag die in familiärer und damit zuverlässiger Runde geführten kritischen Reden unachtsam den falschen Ohren kolportieren könnten. Mit der Angst, die heimlichen heimischen Ausbrüche gegen den totalitären Staat könnten entdeckt werden, korrespondiert auch das folgende Zitat: 
Auch waren wir Profis darin, sooft es darum ging, bei Diskussionen über das Fernsehprogramm des Vorabends entweder den Mund zu halten, wenn der Lehrer ins Zimmer kam, oder statt Wetten dass? und Die versteckte Kamera oder Hart, aber herzlich einfach solche Worte wie Mach mit, mach's nach, mach's besser, Wunschbriefkasten, Ein Kessel Buntes, Inka oder Ralf «Bummi Bursy zu sagen. ${ }^{21}$

Retrospektiv taucht der Topos also im Zusammenhang mit den Eltern auf, wenn es darum geht, die familiäre Gemeinschaft zu DDR-Zeiten zu beschwören - «Unsere Eltern verlangten von uns, dass wir clever waren. $»^{22}-$, um diesen Zusammenhalt später im kulturellen Vergleich mit West-Freunden und -Bekannten effektvoll-kontrastiv in Frage zu stellen: «mit unseren Osteltern blieben wir unabänderlich ein wenig unglücklich.» ${ }^{23}$

\section{Geboren am 13. August ${ }^{24}$}

Mit einem Geburtsdatum vor 1970 ist Jens Bisky ${ }^{25}$ eigentlich zu alt, um zur Generation 89 gezählt zu werden, trotzdem soll seinem Buch Geboren am 13. August hier einige Aufmerksamkeit geschenkt werden, da Bisky in der Süddeutschen Zeitung sowohl eine der ersten wichtigen Rezensionen zu den Zonenkinder veröffentlicht hat, als auch durch Hensels Buch mitangeregt wurde, seine eigene Autobiographie zu schreiben ${ }^{26}$. An Hensels Buch vermisst er das Fehlen einer soziologischen Dimension: «Jana Hensel verzichtet auf Reflexion. Sie scheut die dazugehörige Distanz, schreibt sie doch für alle, die sich wiedererkennen, identifizieren wollen.» ${ }^{27}$

Für Bisky trifft dies nicht zu. Seine alltagsbezogene Naivität aus DDR-Zeiten reflektiert er, als in mehrererlei Hinsicht Betroffener, kritisch in der Rückschau, besonders auch in Bezug auf die Stasi. Für die Zeit vor der Wende heißt es bei ihm:

Die Stasi war mir zu DDR-Zeiten als Spottobjekt vertraut gewesen, Leute, die in auffällig um Unauffälligkeit bemühter Zivilkleidung auf Großveranstaltungen herumstanden, gehörten dazu. [...] In meinen DDR-Tagen schien mir der Spitzelund Überwachungsapparat fern, in einem anderen Land gelegen. Man konnte die Stasi gut verdrängen im Alltagsleben. ${ }^{28}$

Seinen einzigen Kontakt mit dem DDR-Geheimdienst vermutet Bisky während seiner Zeit als Offizier in der NVA. Ein Untergebener hatte verbotenerweise Post aus dem Westen erhalten; diesbezüglich wird er zu einem Vorgesetzten gerufen: «Das kurze Gespräch mit dem Sicherheitsmajor war meine einzige wissentliche Begegnung mit einem MfS-Mitarbeiter, ein unvermeidlicher Dienstkontakt.» ${ }^{29}$ Nach der Wiedervereinigung stellen sich die Tatsachen anders dar. Vom Lehrer über Kommilitonen bis hin zur Mutter ${ }^{30}$ und dem Lebensgefährten nennt Bisky gleich neun ihm bekannte Stasi-Mitarbeiter aus seinem Umfeld.

21 Die Stasi-Tätigkeit des Partners Wolfram hat ihn dabei wohl am stärksten verletzt: «Was Wolfram getan hatte war schändlich, Vertrauensmissbrauch, Verrat»" ${ }^{31}$, und «Das Verbindende, das Innige zwischen Wolfram und mir war geblieben, das Vertrauen stellte sich nicht wieder ein ${ }^{32}$. Bisky beschreibt die Anwerbung Wolframs und die Treff-Routine mit dessen Führungsoffizieren ${ }^{33}$, vor allem aber differenziert er auf der persönlichen und der gesellschaftlichen Ebene die Frage nach schuld und Verantwortung: «Er [Wolfram, M.K.] war IM gewesen, aber doch nicht nur IM, vielen auch einfach ein guter Freund, der durch die Stärke bestach, mit der er lange gegen seine Krankheit gewann.» ${ }^{34}$ 


$$
\text { Grund dieser Erfahrung - auch auf die gesellschaftliche: }
$$

Der Ton in den öffentlichen Debatten missfiel mir. War da nicht ein Grad zu viel an Selbstgerechtigkeit, nicht auch zu viel an schneller Entsorgung nach dem Motto: Wenn alle IMs enttarnt sind, können die ehemaligen Bürger des Landes Frieden mit der Vergangenheit schließen? Ein scharfer Ton herrschte, der verständlich wurde, wenn man sah, wie manche verlogen, feige oder geschickt versuchten, sich $\mathrm{zu}$ rechtfertigen. Geschah in der ungeheuren Aufregung den Opfern der Staatssicherheit Gerechtigkeit? Ich habe kein Recht, für sie zu sprechen, aber es sieht so aus, als hätten sie weder die Aufmerksamkeit noch die Entschädigung erhalten, die ihnen zusteht. ${ }^{35}$

\section{Meine freie deutsche Jugend ${ }^{36}$}

Biskys Beispiel scheint das Erwartete zu bestätigen. Je nach Betroffenheitsgrad der Autorin oder des Autors hält die Stasi-Motivik auch Einzug in das jeweilige Erinnerungsbuch. Für Claudia Ruschs Spiegel-Bestseller, der in 25 lose miteinander verbundenen Geschichten pointiert und tiefsinnig Höhepunkte ihres Lebens in der DDR und der Nachwendezeit erzählt, gilt ähnliches. Wolfgang Hilbig charakterisiert die Texte in seinem Nachwort so:

Die Geschichten von Claudia Rusch sind voller Wärme, voller Solidarität, sie verzichten ganz auf Erfindungen, sie sprechen klar und ohne psychologisches Traktieren vom Innenleben in einem Land, das seinen Leuten nicht gut gesonnen war. ${ }^{37}$

Im Umfeld des DDR-Dissidenten Robert Havemann aufgewachsen, ist sie seit ihrer Kindheit mit dem staatlichen Unterdrückungsapparat aus eigener Anschauung vertraut. So berichtet sie von der Überwachung Havemanns und der Kontrolle seines Hausarrests durch die Stasi ${ }^{38}$, erzählt von der Bespitzelung der Mutter durch den «IM Diamant $\aleph^{39}$, der die Beziehung der Mutter zu ihrem italienischen Freund Claudio, einem KPI-Mitglied, verrät und weiteren Kontakt verhindert, wundert sich in der Erinnerung darüber, dass die Staatssicherheit bereits im Vorfeld von den Reiseplänen der Familie weiß oder beschreibt den Tod ihres Großvater im «Januar 1967 in einer feuchten Zelle der Untersuchungshaftanstalt des MfS in Rostock», ${ }^{40}$ von dem die Familie allerdings erst nach der Wende aus den Stasi-Akten erfährt. Schließlich erzählt sie die Geschichte der - natürlich unbegründeten, für den Spannungsbogen aber notwendigen Verdächtigung der eigenen Großmutter, als vermeintliche «IM Buche» für das MfS tätig gewesen zu sein, hinter der sich dann allerdings pointiert eine langjährige Freundin der Mutter verbirgt, die zuvor über jeden Zweifel erhaben gewesen schien ${ }^{41}$.

Alle Geschichten, selbst die der - im Zuge der Wiedervereinigung eingetretenen StasiHysterie, die in den Medien oft genug den Charakter einer Hexenjagd annahm verdächtigten Großmutter, erzählt Rusch in einer besonderen Weise, mit einer beobachtenden, emotionsarmen, teils spöttischen Distanz. Moralische Wertungen, vielleicht abgesehen vom Fall der «IM Buche», unterbleiben trotz persönlicher Betroffenheit. Ähnlich wie für Bisky, scheint die Stasi aus der Erinnerung an ihr Leben in der DDR eher harmlos gewesen zu sein:

Ich weiß noch, dass ich die Präsenz der Stasi damals nicht wirklich bedrohlich fand.

Für mich waren die ewig wartenden Männer beruhigend. Sie passten auf mich auf.

Ganz im Sinne der Stasi-Ballade: Leibwächter. ${ }^{42}$ 
zei der Geschichten, die in der früheren Kindheit handeln, wird besonders deutlich, wie spielerisch-geschickt Rusch den anekdotischen Wert in Verbindung mit einem naiv-kindlichen Duktus zur Pointierung der Texte nutzt. In «Die Stasi hinter der Küchenspüle» geht es darum, dass ihr als Kind die Mitarbeiter des MfS nur unter der Umschreibung Kakerlaken bekannt waren: «Und weil das so war, wurde ich groß, ohne zu ahnen, was Kakerlaken wirklich sind. Natürlich wusste ich, dass es Küchenschaben gibt, aber ich hatte keinen Schimmer, dass man sie Kakerlaken nennt.» $\gg^{43}$ Als ihr im Alter von 16 Jahren beim Besuch in einem Studentenwohnheim der Gastgeber dann verrät, hinter seiner Küchenspüle hausten rund 200 Kakerlaken, kommt es zu einer komischen Verwechslung, da die Erzählerin natürlich vorgibt, zunächst an Mitarbeiter des MfS und nicht an Schaben gedacht zu haben.

In «Die Hauptabteilung VIII im Märchenwald» ${ }^{44}$ verbindet sich das kindliche mit Märchenmotiven. Auf der Erzählebene aus Sicht der Sechsjährigen imaginiert sich die Erzählerin in eine Märchenwelt zwischen Rotkäppchen und Schneeweißchen und Rosenrot, in der das ihr und ihrer Mutter durch den nächtlichen Wald folgende StasiAuto zur Bedrohung, quasi zum bösen Märchen-Wolf wird. Auf der distanzierten, analytischen Ebene wird dann höchst komisch geschildert, welche Verwirrung es bei den Stasi-Mitarbeitern hervorruft, als die sechsjährige gegen die Angst im Dunkeln ein vielstrophiges NVA-Lied anstimmt und der Dienst-Lada schließlich in einem Schlagloch stecken bleibt:

Es war der Narrenumzug der Saison. Zu NVA-Lied (Nationale Volksarmee) marschierende Tochter vorn, subversive Mutter dahinter, der durchgeschüttelte Stasi-Lada im Schlepptau. Alle in gebührendem Sicherheitsabstand. ${ }^{45}$ Trotz der persönlicher Betroffenheit scheint die Auseinandersetzung bei Rusch hauptsächlich von Distanz gegenüber der Stasi geprägt zu sein. Ähnlich wie bei Bisky wird sie für die Vorwendezeit in ihren alltäglichen Erscheinungsformen als «Spottobjekt» verstanden, woraus kurze, pointenreiche Erzähltexte entstehen.

\section{Ostblöckchen}

Ruschs Geschichten wirken deswegen so treffend, weil sie - anders als in der literarischen Auseinandersetzung direkt nach der Wende - auf moralische Anklagen verzichten, ohne allerdings die Gefährlichkeit und menschenverachtend Vorgehensweise des MfS herunterzuspielen. Statt dessen berichten sie lakonisch und distanziert und wirken dort besonders pointiert, wo kindliche Erzählebene und erwachsene Rückschau aufeinander treffen.

Mit der Form von Michael Tetzlaffs Ostblöckchen ${ }^{46}$ verhält es sich ähnlich. Die kurzen Geschichten, die zum Teil zuvor im Feuilleton der Frankfurter Rundschau erschienen sind, zeichnen sich durch den pointierten, journalistischen Stil aus, der das Anekdotenhafte im Interesse des Leseerlebnisses hervorhebt. Da er, anders als Rusch oder Bisky, allerdings über keine umfangreichen persönlichen Erfahrungen mit dem MfS $\mathrm{zu}$ verfügen scheint, nimmt die Verarbeitung von Stasi-Motiven ausgeprägt komische Züge an. Fast werden die geschilderten Szenen als Burleske vorstellbar, etwa wenn der Vater laut wettert, der Sohn solle nicht zu viel in das Päckchen für die russische Brieffreundin reinpacken: 
«Tu da nicht so viel Klimbim rein, das schnappt sich sowieso alles die Stasi. Dann werden die noch fetter. Die würden sogar die hässlichen Kastanienmännchen fressen, die Brummochsen. Mit den Streichhölzern!»

«Was ist denn Stasi?», fragte ich.

«Die Abkürzung für Starrsinn!», wetterte mein Vater «Ein Verein von Betonköppen, die sich die Hose mit der Kneifzange auziehen und jeden ans Messer liefern, der nicht mindestens genauso blöd und gemein ist wie sie! Merk dir das, ein für alle Mal! Auch das war deutlich.» ${ }^{47}$

31
Klischee der wenig intelligenten, raffgierigen und böswilligen Stasi-Mitarbeiter. Realitätsgetreu kolportiert will die Situation, angesichts der stets drohenden Gefahr des Verrats von systemkritischen Äußerungen, nicht scheinen, zumal auch Tetzlaffs Geschichten - ähnlich wie bei Hensel - Andeutungen auf die allgegenwärtige Gefahr des Verrats unbedachter Äußerungen enthalten, z. B. wenn es um das - verbotene - Westfernsehen geht ${ }^{48}$.

\section{Ausblick}

Natürlich kann die hier vorgestellte Auswahl nicht repräsentativ für das ganze Spektrum der Texte der Generation 89 gelten, zumal etwa die wichtigen Bücher von Peter Richter ${ }^{49}$ oder Jakob Hein ${ }^{50}$ unberücksichtigt geblieben sind ${ }^{51}$. Im Hinblick auf die literarische Auseinandersetzung mit der Stasi lassen sich aber doch einige Rückschlüsse ziehen.

Erwartungsgemäß spiegelt sich die Stasi-Problematik in den Erinnerungsbüchern proportional zur jeweiligen Betroffenheit der Autorin bzw. des Autors. Biskys und Ruschs Bücher sprechen davon. Während Geboren am 13. August- das eher einer herkömmlichen Autobiographie entspricht - allerdings eine stärker reflektierendanalytische Perspektive im Umgang mit der Stasi-Problematik einnimmt ${ }^{52}$, betonen die feuilletonistischen Geschichten aus Meine freie deutsche Jugend das Anekdotenhafte, ohne ins Banale abzugleiten.

Betrachtet man die anderen vorgestellten Texte, scheint sich folgende Tendenz zumindest in Bezug auf die unterhaltsame Tradierung von Klischees und Zuschreibungen zu bestätigen:

Sieht man von Nachzüglern ab wie Michael Tetzlaff [...] oder Peter Richter [...], plagen sich junge, in der DDR geborene Autoren und Autorinnen immer weniger mit Identitätsproblematik herum als vielmehr damit, vordergründig die verlorene Vergangenheit zu archivieren - sie spielen höchstens noch kokett mit Klischees und Zuschreibungen. ${ }^{53}$

Die literarische Suche nach eigener Identität steht sowohl bei Hensel als auch bei Tetzlaff im Vordergrund; während sich die eine in der Nachwendezeit zurecht finden muss, sucht der andere Spuren seiner Herkunft in der thüringischen Provinz. Motive im Zusammenhang mit der Stasi tauchen hier allerdings nur noch zur humorvollen Befriedigung von Erwartungen und Klischees auf, so hat es den Anschein. 


\section{NOTES}

1. Michael Tetzlaff, Ostblöckchen. aus der Zone, Frankfurt a. M.: Schöffling \& Co 2004, S. 105. - Ein umfassender Überblick über die Literatur zur Deutschen Einheit einschließlich Generation 89 findet sich auf den Seiten des Goethe-Instituts Bordeaux:http://www.goethe.de/ins/fr/bor/pro/ wende/d_menue.htm [zuletzt besucht am 31. 5. 2006].

2. Ingo Schulze, Simple Storys. Roman aus der ostdeutschen Provinz, München: $\mathrm{dtv}^{3} 2001$ (1998). Ingo Schulze (geb. am 15. Dezember 1962 in Dresden) studierte nach dem Abitur von 1983 bis 1988 Klassische Philologie an der Universität Jena, danach bis 1990 Tätigkeit als Dramaturg am Landestheater in Altenburg/Thüringen. Im Anschluss Arbeit als Jounalist und Redakteur, außerdem ein halbjähriger Aufenthalt in St. Petersburg, Russland. Ingo Schulze wurde mit dem Alfred-Döblin-Förderpreis, dem Aspekte-Literaturpreis, der Johannes-Bobrowski-Medaille u.a. ausgezeichnet. Weitere Werk (auswahl): 33 Augenblicke des Glücks, Berlin-Verlag 1995; Neue Leben, Berlin: Berlin Verlag 2005.

3. Christoph Hein, Willenbrock, Frankfurt a. M.: Suhrkamp 2000.

4. Wolfgang Höbel, Glücksritter auf Tauchstation. In: Der Spiegel, 10/1998, S. 218.

5. Schulze, a. a. A., S. 21.

6. Uwe Wittstock: «Ein Herz für die Herzlosen. Der Romancier Christoph Hein erzählt vom reichen Leben ohne Utopie». In: Die Welt vom 27. 1. 2001.

7. Christoph Hein, a. a. A., S. 106.

8. Ebd., S. 246-250.

9. Ebd, S. 250.

10. Schulze, a. a. A., S. 33.

11. Jana Hensel, Zonenkinder, Reinbek bei Hamburg: Rowohlt 2002 ( $\left.{ }^{1} 2001\right)$. Jana Hensel (geb. 1976 in Leipzig) studierte in Leipzig, Marseille, Berlin und Paris. Anschließend war sie von 1999 Herausgeberin der Leipziger Literaturzeitschrift EDIT; die Autorin lebt derzeit in Berlin und schreibt u. a. für Die Zeit. Siehe auch: Dies. u. Thomas Hettche: Null, Internet-Anthologie, Köln: DuMont 2000.

12. Florian Illies, Generation Golf. Eine Inspektion, Frankfurt a. M.: Fischer 2001.

13. Hensel, a. a. A., S. 166.

14. Karl Heinz Bohrer: «Die DDR als eine Art Naturschutzpark». In: $F A Z$ vom 13. Januar 1990.

15. Ebd.

16. Gesprochen mit Karl Rosenkranz: Ästhetik des Hässlichen, Leipzig: Reclam ${ }^{2} 1996$ [ $\left.{ }^{1} 1853\right]$.

17. Susanne Messmer: «Unsere schönen Projektionen». In: taz, Nr. 6924, 7.12.2002, S. 13.

18. Zit. n. Robin Alexander: «Die vergoldete Zonenjugend. Sie sind jung. Sie sind erfolgreich. Sie treffen sich in einem Schwimmbad ohne Wasser. Die Generation 89 ist da. Na ja. »In: taz, Nr. 7196, 31.10.2003, S. 16.

19. Tom Kraushaar (Hg), Die Zonenkinder. Die Geschichte eines Phänomens, Reinbek bei Hamburg: Rowohlt 2004, S. 25.

20. Hensel, Zonenkinder, S. 78.

21. Ebd., S. 90.

22. Ebd.

23. Ebd., S. 69.

24. Jens Bisky, Geboren am 13. August. Der Sozialismus und ich, Berlin: Rowohlt 2004.

25. Jens Bisky wurde 1966 als ältester Sohn des heutigen Vorsitzenden der Linkspartei-PDS Lothar Bisky geboren, war nach dem Abitur Unterleutnant in der NVA, arbeitete zur Wendezeit bei «Jugendradio DT 64», studierte anschließend Kulturwissenschaften und Germanistik, arbeitete für die Berliner Zeitung und ist heute Feuilleton-Redakteur der Süddeutschen Zeitung. 
26. Die Anekdote dazu findet sich bei Christoph Amend, «Diese Biskys. Vater Lothar ist Chef der PDS, Sohn Norbert erfolgreicher Maler, Mutter Almuth war IM - und Sohn Jens hat über alles ein Buch geschrieben. Porträt einer deutschen Familie». In: Die Zeit vom 23.9.2004.

27. Jens Bisky, «Traumbilder vom Osten in den Farben des Westens», in: Tom Kraushaar (Hg.), Die Zonenkinder. Die Geschichte eines Phänomens, Reinbek bei Hamburg: Rowohlt 2004, S. 28. Zuerst in: Süddeutsche Zeitung vom 9.10.2002.

28. Bisky, a. a. O., S. 237.

29. Ebd.

30. Jens Biskys Mutter Almuth war zu DDR-Zeiten Kulturfunktionären und bespitzelte als IM u. a. Erich Loest. (Vgl. Amend, a. a. O.).

31. Bisky, a. a. O., S. 234.

32. Ebd., S. 236.

33. Vgl. ebd., S. 234-235.

34. Ebd., S.236-237. Es handelt sich aller Wahrscheinlichkeit nach um den Drehbuchautor Wolfram Witt (*20.05.1953 Weimar, $\uparrow 19.08 .2003$ Berlin), der zusammen mit Heiner Carow (Regie) Coming out (1988/89) und Die Verfehlung (1991) gedreht hat.

35. Ebd., S. 238.

36. Claudia Rusch, Meine freie deutsche Jugend, Frankfurt a. M.: Fischer 2005 ( $\left.{ }^{1} 2003\right)$. Claudia Rusch, geboren 1971 in Stralsund, wuchs auf Rügen und in Brandenburg auf, machte 1990 ihr Abitur in Berlin; danach Studium der Germanistik und Romanistik in Berlin, Bologna und Paris. Anschließend arbeitete sie sechs Jahre als Fernsehredakteurin; seit 2001 lebt sie als freie Autorin in Berlin.

37. Wolfgang Hilbig: «Claudia Ruschs, Meine freie deutsche Jugend». In: Rusch, Meine freie deutsche Jugend, S. 155.

38. Rusch, Meine freie deutsche Jugend, S. 16-17 u. 22-24.

39. Ebd., S. 34.

40. Ebd., S. 109.

41. Ebd., S. 111-117.

42. Ebd., S. 16-17; die Stasi-Ballade Leibwächter ist von Wolf Biermann (1967).

43. Ebd., S. 17.

44. Die Hauptabteilung VII war innerhalb des MfS für die «Abwehr » staatsfeindlicher Umtriebe innerhalb der DDR zuständig.

45. Ebd., S. 24.

46. Michael Tetzlaff, geboren 1973 in Eisenberg, wuchs in Thüringen auf; heute lebt und arbeitet er in Frankfurt a. M.

47. Ebd., S. 105.

48. Vgl. ebd., S. 110-111.

49. Peter Richter, Blühende Landschaften. Eine Heimatkunde, München: Goldmann 2004. Peter Richter, 1973 in Dresden geboren und dort auch aufgewachsen, studierte nach der Wende Kunstgeschichte in Hamburg, arbeitete als Autor für die Süddeutsche Zeitung, war Redakteur bei der Frankfurter Allgemeinen Zeitung, lebt in Berlin und ist als Kolumnist für die Frankfurter Allgemeine Sonntagszeitung tätig.

50. Jakob Hein, Mein erstes T-Shirt. Mit einem Vorwort von Wladimir Kaminer, München: Piper ${ }^{5} 2005$ (12003). Jakob Hein wurde 1971 als Sohn des Autors Christoph Hein geboren. Nach dem Abitur 1990 studierte er Medizin in Berlin und Boston, promovierte 2000 an der HumboldtUniversität Berlin und arbeitet heute als Arzt in der Kinderpsychiatrie an der Berliner Charité und als Schriftsteller. Weitere Werke: Formen menschlichen Zusammenlebens, München: Piper 2003; Vielleicht ist es sogar schön, München: Piper 2004, Mexiko - Mexiko. Mit vier farbigen Originallinolschnitten, Berlin: Berliner Handpresse 2005 und Herr Jensen steigt aus, München: Piper 2006. 
51. Zur Frage etwa der Komik in den Texten zur Wiedervereinigung vgl. Oliver Igel: Gab es die DDR wirklich? Die Darstellung des SED-Staates in komischer Prosa zur «Wende», Tönning, Lübeck u. Marburg: Der Andere Verlag 2005.

52. Diesbezüglich liegt die Vermutung nahe, dass Biskys Alter eine Rolle spielt.

53. Susanne Messmer: «Die neuen Zonenkinder. Junge, noch in der DDR geborene Autoren und Autorinnen plagen sich nicht mit deutsch-deutscher Identitätsproblematik herum, sondern stellen das Originelle und Spezifische ins Zentrum ihres Schreibens». In: taz, vom 28. 5. 2004, S. 15.

\section{RÉSUMÉS}

In der erzählenden Literatur zur Deutschen Einheit nimmt die Auseinandersetzung mit dem ehemaligen Ministerium für Staatssicherheit der DDR, der «Stasi», und verwandter Motive breiten Raum ein. Folgender Artikel untersucht an Texten von Jana Hensel, Jens Bisky, Claudia Rusch und Michael Tetzlaff, ob und inwieweit dies auch für die «Generation 89» gilt, also jene Generation von noch in der DDR geborenen und im vereinten Deutschland aufgewachsenen Autorinnen und Autoren, die mit ihren Erinnerungsbüchern zu Publikumserfolgen geworden sind.

Dans les récits littéraires ayant pour objet l'unification allemande, le débat autour de l'ancien ministère de la Sécurité, la "Stasi », et les thèmes qui y sont associés, occupent une place importante. Dans notre étude, nous analyserons à la lumière des textes de Jana Hensel, Jens Bisky, Claudia Rusch et Michel Tetzlaff, comment et dans quelle mesure cela se vérifie aussi pour la génération 89 , cette génération d'écrivains de RDA qui a grandi dans l'Allemange réunifiée et dont les livres de souvenirs sont devenus des best-sellers.

\section{INDEX}

Mots-clés : RDA, Stasi, unification allemande

oeuvrecitee Blühende Landschaften, geboren am 13 - August - Der Sozialismus und ich, Mein freie deutsche Jugend, Ostblöckchen, Zonenkinder, Mein erstes T-shirt

\section{AUTEURS}

MICHAEL KLEES

Gießen 